\title{
The validation of the PRISMA-7 questionnaire in community-dwelling elderly people living in Antalya,
} Turkey

\author{
Hakan Yaman ${ }^{1}$, Zeynep Ünal ${ }^{2}$
}

${ }^{1}$ MD in Family Medicine, Former Professor of Akdeniz University, Konyaalt1- Antalya, Turkey

${ }^{2}$ MSC of Bioinformatics; PhD Candidate in Econometrics, Institute of Social Sciences, Akdeniz University, Antalya, Turkey

\section{Type of article: Original}

\begin{abstract}
Background: The screening of frailty in older adults is an important issue in the preventive approach in medicine. Frailty is one of the leading causes of morbidity and premature mortality in older adults.

Objective: The aim of this study was to validate the PRISMA-7 questionnaire in community-dwelling elderly people living in Antalya, Turkey.

Methods: This study was cross-sectional and observational in nature and was conducted in Antalya (Turkey) from February 2016 to April 2017. One hundred fifty patients participated in this study. PRISMA-7 and the CSHA Clinical Frailty Scale were applied to these patients along with a questionnaire on socio-demographics. No translations were available for the PRISMA-7 or the CSHA in Turkish language. The PRISMA-7 and the CSHA Clinical Frailty Scale were translated by two translators to Turkish language; after compilation and agreeing to one Turkish translation, the text was translated back by two translators to English. The translation was then compiled to one text and compared with the original text. After agreement on the translation, both translations were piloted in 10 elderly people. The final form has been applied in this study. Data of the ten piloted patients have not been included into the dataset. Data were analyzed by IBM-SPSS version 22, using descriptive statistics, Pearson product-moment correlation, and receiver operator characteristic (ROC) analysis. The level of significance was set at 0.05 .

Results: Our study showed that participants were in average vulnerability to frailty. The test-retest reliability score for the PRISMA-7 Scale and for the CSHA Clinical Frailty Scale were $(r=99.2 ; p<0.001)$ and $(r=97.5$; $\mathrm{p}<0.05$ ), respectively. After using CSHA Clinical Frailty Scale as a reference standard, PRISMA-7 showed that the area under ROC curve (AUC) was 0.903; which is the best accuracy; and medium level for internal consistency (Cronbach's $\alpha=0.714$ ) as a measure for reliability. A cut-off point of 4 or higher for PRISMA7 revealed high sensitivity $(81.5 \%)$ and specificity $(88.2 \%)$ for frailty.

Conclusion: We would recommend PRISMA-7 as a reliable and valid instrument, with high-level accuracy in the screening process of frailty.

Keywords: Aging, Frailty, Geriatric Assessment, Disability Evaluation, Turkey
\end{abstract}

\section{Introduction}

Frailty is a syndrome that results in worse outcomes (1), and needs an appropriate diagnostic approach in elderly people. It is a condition that comprises daily coping mechanisms and functionality due to loss of body reserves and capabilities (2). Therefore, an early diagnosis of the pre-frail state is warranted to determine people who are prone to frailty and to intervene properly to overcome the "vicious cycle" of frailty. The progression to this condition could be stopped or ameliorated (1). From an epidemiological point of view, an increase of its frequency could be

\section{Corresponding author:}

Professor Dr. Hakan Yaman, Uncalı Mh. 1262. Sk. No: 15, Oksuzoglu Konakları, A Blok Kat: 2 Daire 5, Konyaaltı, 07070 Antalya-Turkey, Tel: 009053632099 33, Email: hakanyam@yahoo.com

Received: May 28, 2018, Accepted: June 23, 2018, Published: September 2018

iThenticate screening: June 14, 2018, English editing: August 01, 2018, Quality control: August 12, 2018

This article has been reviewed / commented by three experts

Ethics approval: 70904504/205 (Akdeniz University, Date: May 13, 2015)

(C) 2018 The Authors. This is an open access article under the terms of the Creative Commons Attribution-NonCommercialNoDerivs License, which permits use and distribution in any medium, provided the original work is properly cited, the use is non-commercial and no modifications or adaptations are made. 
observed. Lack of a gold standard is still an obstacle for the definition of frailty. Therefore, a clear epidemiological picture is not yet evident (3). There are two measures widely used for the definition of frailty in literature: the Fried criteria (2) and the Frailty Index. The Frailty Index is a count of 70 clinical deficits from the clinical assessment of the Canadian Study of Health and Aging (CSHA) (4). It is estimated that in a community, the prevalence of frailty is estimated at between 4 and $59.1 \%$ (3). According to the Fried criteria, the prevalence of frailty was $9.9 \%$ and of prefrailty $44.2 \%(2,5)$. Whereas the Frailty Index from the CSHA showed an increase of prevalence with aging (6), studies from Turkey showed different results as well (7). A community-based study reported frailty, pre-frailty and non-frailty prevalence of $27.8,34.8$, and $37.4 \%$ according to the Fried Frailty Index, respectively (8). A sample from an academic and training hospital-based outpatient survey showed a prevalence of frailty and pre-frailty of $39.2 \%$ and $43.3 \%$ with modified Fried criteria, respectively (9). Family practice is in an especially good position to identify frail patients in community. A pro-active preventive approach is the key to finding cases at risk and to intervene on these. The diagnosis of frailty is mainly based on the clinical judgment of the patient by the physician. Questionnaires, scales, patient complaints or the information from relatives are taken into consideration. For a reliable and objective identification of frailty in patients, well-validated and reliable scales are needed (10). These scales or instruments must fit the needs of family physicians. A comprehensive geriatric assessment is mostly not practicable in family practice, because of time-demands, low resources and heavy demand to patients (11). A continuing effort is spent to solve this obstacle (12). This is the case also for Turkey. The concept of frailty screening in Turkey is relatively new and there is an urgent need for a short, less-time-consuming measure, which could be easily implemented in daily, busy practice. We chose therefore, the PRISMA 7 Questionnaire, which is a test comprising of seven questions and was recommended by the British Geriatric Society as a first instance screening test $(13,14)$. The aim of this study was to validate the PRISMA-7 questionnaire in community-dwelling elderly people living in Antalya, Turkey.

\section{Material and Methods}

\subsection{Setting and design}

The study was cross-sectional and observational in nature. It was conducted in Antalya, Turkey, from February 2016 to April 2017. Patients involved in this study were asked either during home visit or during an outpatient encounter to participate in this study.

\subsection{Selection criteria}

\subsubsection{Inclusion criteria}

The following were set as the inclusion criteria: age $\geqslant 65$ years, being able to communicate and answer the questionnaire items, being in good cognitive state, and completing all instruments and scales. Cognitive state was determined clinically by the medical staff of the home health care or outpatient care team.

\subsubsection{Exclusion criteria}

The exclusion criteria were the following: Age $<65$ year, being unable to communicate or answer the questionnaire items, being in worse cognitive state, and incomplete instruments and scales.

\subsection{Sampling}

\subsubsection{Sample size}

One hundred fifty patients participated in this study. The sample size was calculated for a prevalence of $11 \%$, margin of error $=5 \%$, and confidence level of $95 \%$.

\subsubsection{Sampling method}

Since the aim of this study was not an epidemiologic and community-based one, the purposive sampling method on of consecutively visited or attending participants was used in the study. Heterogeneous sampling was performed, where different ranges of cases relevant to potential frailty were selected.

\subsection{Instruments}

The PRISMA-7 and the CSHA Clinical Frailty Scale were applied to these patients along with a questionnaire on socio-demographics (i.e. age, sex, marital status, education, and income) and social and functional status (i.e. meeting relatives, assistance, loneliness, physical aid, and physical assistance). Permission was granted from the authors of PRISMA-7. The PRISMA-7 is a questionnaire with seven items. Items consist of domains such as age $>85$ years, male gender, health problems that limit activities, need for support by others, health problems that require staying at home, having someone to count on, use of stick or walker or wheelchair. Each positively answered item revealed one point. A score of three or more indicates frailty and warrants a further evaluation. This questionnaire was found to be relatively sensitive after comparing with the Système de Mesure de l'Autonomie Fonctionnelle 
scale (SMAF) (sensitivity $=0.78$ and specificity $=0.74$ (14-16). PRISMA-7 showed the best accuracy after using Fried's frailty criteria as a reference standard (Area under curve: AUC $=0.85)(14,17)$. The CSHA Clinical Frailty Scale is an effective instrument, which has domains (Very Fit, Well, Managing Well, Vulnerable, Mildly Frail, Moderately Frail, Severely Frail, Very Severely Frail, and Terminally Ill). This scale showed good criterion validity (5-year mortality prediction and institutionalizing), good construct validity (poor health outcomes), and it has been better than the rules-based frailty definition. The CSHA Clinical Frailty Scale and Frailty Index (mathematically derived) had high construct validity $(\mathrm{r}=0.80, \mathrm{p}<0.01)$ (4). Patients were evaluated with the CSHA Clinical Frailty Scale and frailty was defined as a score of 5 or higher (18). No translation was available for the PRISMA-7 or the CSHA in Turkish language. The PRISMA-7 and the CSHA Clinical Frailty Scale were translated by two translators to Turkish language; after compilation and agreeing to one Turkish translation, the text was translated back to English by two translators. The translation was compiled to one text and compared with the original text. After agreement on the translation, both translations were piloted in 10 elderly people. Final form has been applied in this study. Data of the ten piloted patients have not been included into the dataset.

Face validity was determined by two experts in this field. Construct validity was determined by factor analysis with principal component extraction method. All seven items were included into two components (Table 1). A recent linguistic validation study of PRISMA-7 showed similar two-component factor structure and items 2 and 6 were similarly forming the second component (19). Exclusion of item 6 ("In case of need, can you count on someone close to you?") resulted in one component factor structure, but only $48 \%$ of the total was explained. Therefore, the two-component factor structure was accepted. Reliability was determined by internal consistency and test-retest reliability measurement. A high proportion of highest or lowest possible values challenges the differentiation of participants from each other and causes low reliability. This phenomenon is called floor and ceiling effect. These effects are observed when more than $15 \%$ of measurements are within these values. A sample size of at least 50 participants without any floor and ceiling effect can be used for content validity analysis (20). In our study, only 4.7\% (7/150) of respondents had highest or lowest possible numeric values in the PRISMA-7. Therefore, no floor or ceiling effect could be observed in our dataset.

Table 1. Factor structure of PRISMA-7

\begin{tabular}{|l|l|l|}
\hline \multicolumn{3}{|l|}{ Rotated Component Matrix } \\
\hline & \multicolumn{2}{l|}{ Component } \\
\cline { 2 - 3 } & 1 & 2 \\
\hline Prisma5 & 0.896 & \\
\hline Prisma3 & 0.886 & \\
\hline Prisma4 & 0.862 & \\
\hline Prisma7 & 0.707 & \\
\hline Prisma1 & 0.512 & \\
\hline Prisma2 & & 0.732 \\
\hline Prisma6 & & 0.650 \\
\hline
\end{tabular}

a: Rotation converged in 3 iterations. Extraction Method: Principal Component Analysis.

Rotation Method: Varimax with Kaiser Normalization

\subsection{Research ethics}

This study is a part of a comprehensive study covering six specialization theses, and was approved in 2015 (Ref: 70904504/205, Akdeniz University Faculty of Medicine Ethical Committee; date: 13/05/2015). Data on PRISMA-7 and CHSA scale were collected subsequently in the setting mentioned in this paper. Informed consent was obtained from all individual participants and all procedures performed in studies involving human participants were in accordance with the ethical standards of the institutional and/or national research committee and with the 1964 Helsinki declaration and its later amendments or comparable ethical standards.

\subsection{Statistical analysis}

Data were analyzed by IBM $\odot$ SPSS $\odot$ Statistics version 22 (IBMC Corp., Armonk, NY, USA), using descriptive analysis, internal consistency (Cronbach's alpha score), test-retest reliability examination, Pearson product-moment correlation, and ROC analysis. Using obtained state variables "Frail" and "Not Frail," receiver operator characteristic (ROC) curves were generated and area under the ROC curve (AUC) were used for study outcomes of the frailty scales. The AUC ranges from 0.5 to 1.0 , where 1.0 indicates perfect sensitivity and specificity. An index 
test AUC of at least 0.8 is considered to indicate good discriminative ability. The level of significance was set at 0.05 .

\section{Results}

One hundred-fifty participants participated in this study (participation rate: 95\%). Most of the participants were women $(\mathrm{n}=95,63 \%)$ while $55(37 \%)$ were men. Their mean age was 76.8 years $(\mathrm{SD}=8.2$ years, min-max $=65-104$, $\mathrm{n}=150$ ). Their sociodemographic characteristics are shown in Table 2. Also, findings on the conditions concerning the social and functional status of participants are shown in Figure 1. Results of PRISMA-7 and CSHA Clinical Frailty Scale are shown in Table 3. The data of a subgroup of 30 participants (age $=72.67, \mathrm{SD}=6.12$, $\min$ - $\max =65$ $85 ; n=18,60 \%$ women and $n=12,40 \%$ men) were used for subgroup analysis (Table 3 ). The test-retest reliability score for the PRISMA-7 Scale and for the CSHA Clinical Frailty Scale were $(r=99.2 ; p<0.001)$ and $(r=97.5$; $\mathrm{p}<0.05$ ), respectively. Using The CSHA Clinical Frailty Scale as a reference (gold) standard, PRISMA-7 showed the best accuracy (AUC $=0.903$ ). Cronbach's $\alpha$ coefficient was 0.714 for the PRISMA-7 scale. Intraclass Correlation for single measures and for average measures were $0.263(\mathrm{p}<0.001)$ and $0.714(\mathrm{p}<0.001)$, respectively. The area under the ROC curve (AUC) for the PRISMA-7 scale versus the CSHA Clinical Frailty Scale was 0.903 (Figure 2). A cutoff point of 4 or higher for PRISMA-7 was applied, and indicated the sensitivity of $81.5 \%$, and specificity of $88.2 \%$ (Table 4).

Table 2. Sociodemographic characteristics of the participants $(\mathrm{n}=150)$

\begin{tabular}{|l|l|l|}
\hline Variables & Female & $\mathrm{n}(\%)$ \\
\hline \multirow{4}{*}{ Sex } & Male & $95(63)$ \\
\hline \multirow{4}{*}{ Marital Satus $(76.8 \pm 8$ year) } & $65-74$ & $55(37)$ \\
\cline { 2 - 3 } & $75-84$ & $64(44)$ \\
\cline { 2 - 3 } & $\geq 85$ & $56(37)$ \\
\hline \multirow{5}{*}{ Educational level } & Married & $30(20)$ \\
\cline { 2 - 3 } & Widowed & $74(49)$ \\
\cline { 2 - 3 } & Single & $63(42)$ \\
\cline { 2 - 3 } & Divorced & $9(6)$ \\
\hline & Illiterate & $4(3)$ \\
\cline { 2 - 3 } & Literate & $32(21)$ \\
\cline { 2 - 3 } & Primary school & $45(30)$ \\
\cline { 2 - 3 } & Secondary school & $4(36)$ \\
\cline { 2 - 3 } & High school & $11(7)$ \\
\cline { 2 - 3 } & University & $4(3)$ \\
\hline Income and spending of participants & Income = spending & $97(65)$ \\
\cline { 2 - 3 } & Income < spending & $45(30)$ \\
\cline { 2 - 3 } & Income $>$ spending & $8(5)$ \\
\hline
\end{tabular}

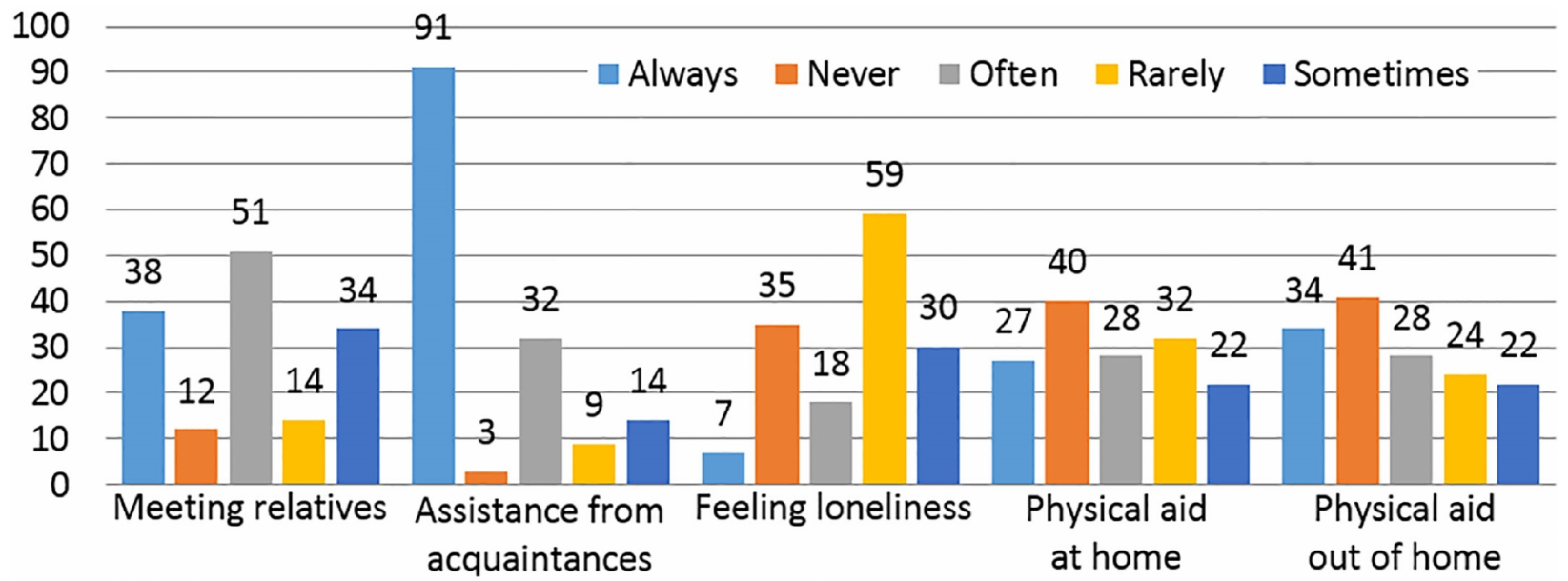

Figure 1. Conditions concerning the social and functional status of participants 
Table 3. Frailty measures for the whole sample $(n=150)$

\begin{tabular}{|l|l|l|l|l|}
\hline \multicolumn{2}{|l|}{ Frailty Measures } & Mean & SD & Min-Max \\
\hline whole sample $(\mathrm{n}=150)$ & The PRISMA-7 & 3.07 & 1.89 & $0-7$ \\
\cline { 2 - 5 } & The CSHA Clinical Frailty Scale & 4.41 & 1.757 & $1-7$ \\
\hline \multirow{2}{*}{ the subgroup sample $(\mathrm{n}=30)$} & The PRISMA-7 & 2.40 & 1.77 & $0-7$ \\
\cline { 2 - 6 } & The CSHA Clinical Frailty Scale & 3.33 & 1.67 & $1-7$ \\
\hline
\end{tabular}

Table 4. PRISMA-7 Cut-Off Values

\begin{tabular}{|l|l|l|l|l|}
\hline Cut-off & Sensitivity (\%) & Specificity (\%) & $\begin{array}{l}\text { Positive Predictive } \\
\text { Value (\%) }\end{array}$ & $\begin{array}{l}\text { Negative Predictive } \\
\text { Value (\%) }\end{array}$ \\
\hline 0 & 100.0 & 0.0 & 43.3 & \\
\hline 1 & 100.0 & 4.7 & 44.5 & 100.0 \\
\hline 2 & 93.8 & 44.7 & 56.5 & 90.5 \\
\hline 3 & 87.7 & 76.5 & 74.0 & 89.0 \\
\hline 4 & 81.5 & 88.3 & 84.1 & 86.2 \\
\hline 5 & 64.6 & 97.6 & 95.5 & 78.3 \\
\hline 6 & 30.8 & 100.0 & 100.0 & 65.4 \\
\hline 7 & 4.6 & 100.0 & 100.0 & 57.8 \\
\hline
\end{tabular}

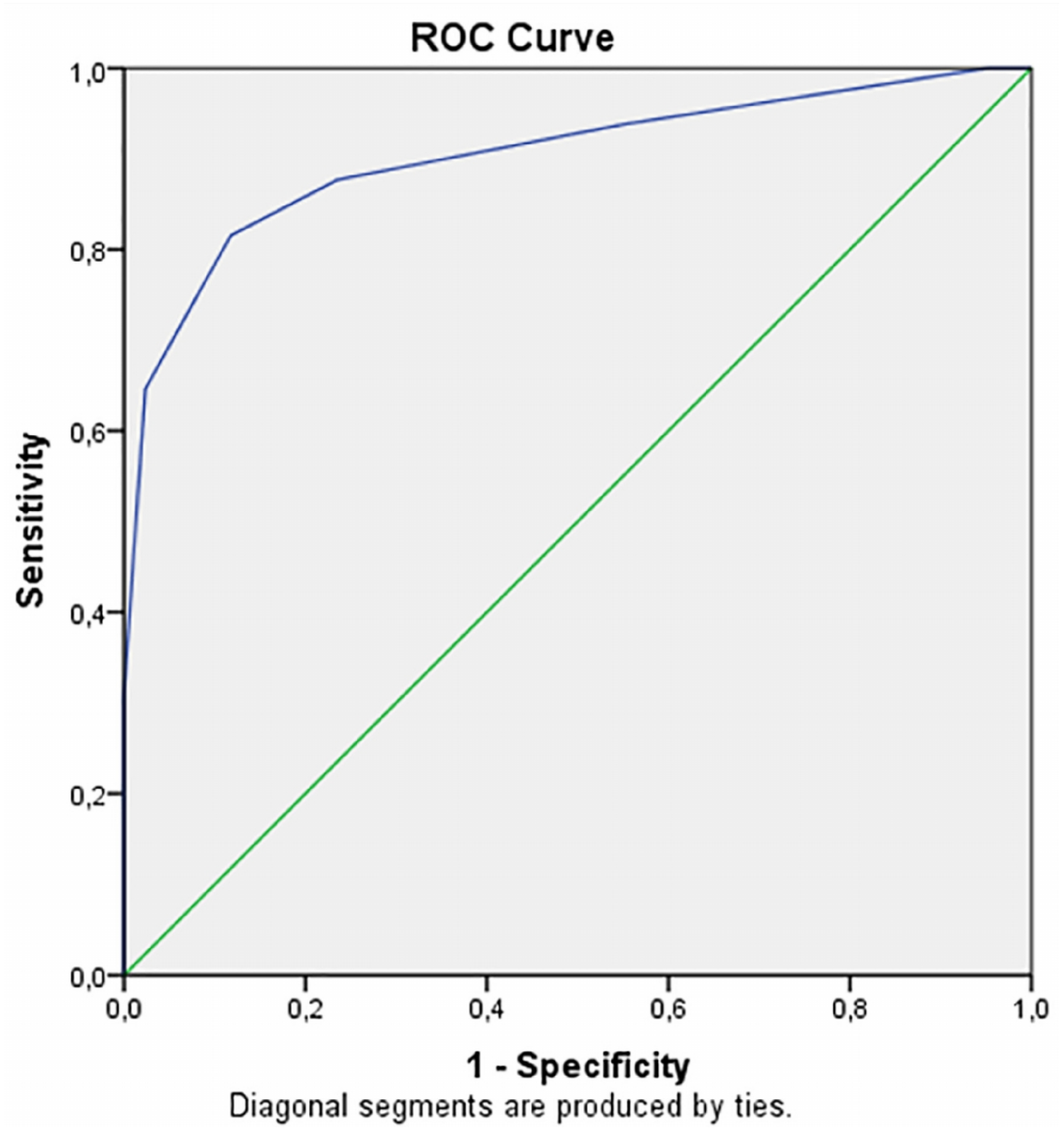

Figure 2. PRISMA-7 ROC Curve

\section{Discussion}

The aim of this study was to validate the PRISMA-7 questionnaire in community-dwelling elderly people living in Antalya, Turkey. They were at medium level connected to their relatives, had high level of support in case of an emergency, rarely felt lonely and had medium level assistance at home and outside. Higher level of frailty, low-level 
involvement in social leisure activities and low functional status were shown to have a strong relationship with decreased subjective well-being (15). Another study found an association with age, functional status and nutritional status with frailty (16). Men were associated with non-white-collar jobs, inadequate financial management, less sportive activities, no alcohol, low social network, and less involvement in supportive activities to others. Women had additionally low social contact with relatives and involvement community activities factors predicting frailty (17). In general, walking difficulties, need of a supportive walking device, need of caregiver support, depression, and functional dependence (instrumental activities of daily living) were related with frailty (18). Our study revealed medium level meeting with relatives, high level assistance in case of an emergency situation, rarely feeling loneliness, medium level having a physical aid at home, and medium level physical assistance outside of home. Our patients were in average apparently vulnerable stage, which is stage four of nine stages ( $1=$ very fit, $9=$ terminally ill) of the CSHA Clinical Frailty Scale. Looking at test-retest, reliability performance of both tests was very high. The PRISMA-7 scale was developed in Canada and showed ability to detect elderly people, who suffer Frailty (14, 19). A cut-off score of three and above positive answers to a total seven questions revealed a sensitivity of $78.3 \%$ and a specificity of $74.7 \%$, which might identify $35.5 \%$ of the aged people as frail. Increasing the threshold to four or above showed a sensitivity of $60.9 \%$ and a specificity of $91 \%$ (19\% frail) (19). The Brazilian translation showed borderline internal consistency. Factor analysis retrieved two well-correlated factors (except items 2 and 6) (20). In their study, ROC curve analysis showed sensitivity of $74.4 \%$ and specificity of $87.4 \%$ for cut-off point of 4 (20). In our study, we used the CSHA Clinical Frailty Scale as a reference standard. PRISMA-7 showed the best accuracy $(\mathrm{AUC}=0.903$ ) with this standard. The PRISMA-7 scale had an acceptable internal reliability (Cronbach's coefficient $\alpha=0.714$. A cut-off point of 4 or higher for PRISMA-7, revealed high sensitivity (81.5\%) and specificity (88.2\%) for frailty. This was in congruence with the Brazilian version (20) and 1 score higher than the Canadian version (14, 19).

\section{Strength and limitations}

The strength of this study is its high sensitivity and specificity. The method of sampling is minimizing its generalizability to the whole population. But the purposeful sample is still relevant in giving an opinion in the frail Turkish population.

\section{Conclusions}

In summary, the study showed a high level of test-retest reliability of the PRISMA-7 and CSHA Clinical Frailty Scales. After using CSHA Clinical Frailty Scale as a reference standard, PRISMA-7 showed the best accuracy and medium level internal reliability. A cut-off point of 4 or higher for PRISMA-7, revealed high sensitivity and specificity for frailty. We would recommend PRISMA-7 as a reliable and valid instrument, with high level accuracy in the screening process of frailty in Turkey.

\section{Acknowledgments:}

The authors are grateful for the support of the Special Study Module Students at Akdeniz University, Faculty of Medicine.

\section{Conflict of Interest:}

There is no conflict of interest to be declared.

Authors' contributions:

Conception - HY, ZÜ; Design - HY,ZÜ; Review - HY,ZÜ; Data Collection - HY,ZÜ; Analysis - ZÜ; Literature search - HY,ZÜ; Writing - HY,ZÜ; Final approval - HY,ZÜ.

\section{References:}

1) Xue QL. The frailty syndrome: definition and natural history. Clin Geriatr Med. 2011; 27(1): 1-15. doi: 10.1016/j.cger.2010.08.009. Review. PMID: 21093718, PMCID: PMC3028599.

2) Fried LP, Tangen CM, Walston J, Newman AB, Hirsch C, Gottdiener J, et al. Frailty in older adults: evidence for a phenotype. J Gerontol A Biol Sci Med Sci. 2001; 56(3): M146-56. PMID: 11253156.

3) Collard RM, Boter H, Schoevers RA, Oude Voshaar RC. Prevalence of frailty in community-dwelling older persons: a systematic review. J Am Geriatr Soc. 2012; 60: 1487-92. doi: 10.1111/j.15325415.2012.04054.x. PMID: 22881367. 
4) Rockwood K, Song X, MacKnight C, Bergman H, Hogan DB, McDowell I, et al. A global clinical measure of fitness and frailty in elderly people. CMAJ. 2005; 173(5): 489-95. PMID: 16129869, PMCID: PMC1188185.

5) Cesari M, Prince M, Thiyagarajan JA, De Carvalho IA, Bernabei R, Chan P, et al. Frailty: An Emerging Public Health Priority. J Am Med Dir Assoc. 2016; 17(3): 188-92. doi: 10.1016/j.jamda.2015.12.016. PMID: 26805753.

6) Mitnitski A, Rockwood K. The rate of aging: the rate of deficit accumulation does not change over the adult life span. Biogerontology. 2016; 17(1): 199-204. doi: 10.1007/s10522-015-9583-y. PMID: 25972341.

7) Yaman H. Frailty in Family Practice: A Survey of Caregivers of Frail Elders. Middle East Journal of Age and Aging . 2017; 14(3): 3-9. doi: 10.5742/MEJAA.2017.93090.

8) Akın S, Mazıcıoglu MM, Mucuk S, Gocer S, Deniz Şafak E, Arguvanlı S, et al. The prevalence of frailty and related factors in community-dwelling Turkish elderly according to modified Fried Frailty Index and FRAIL scales. Aging Clin Exp Res. 2015; 27(5): 703-9. doi: 10.1007/s40520-015-0337-0. PMID: 25762157.

9) Eyigor S, Kutsal YG, Duran E, Huner B, Paker N, Durmus B, et al. Frailty prevalence and related factors in the older adult-FrailTURK Project. Age (Dordr). 2015; 37(3): 9791. doi: 10.1007/s11357-015-9791-z. PMID: 25948502, PMCID: PMC4422824.

10) Sutorius FL, Hoogendijk EO, Prins BA, van Hout HP. Comparison of 10 single and stepped methods to identify frail older persons in primary care: diagnostic and prognostic accuracy. BMC Fam Pract. 2016; 17: 102. doi:10.1186/s12875-016-0487-y. PMID: 27488562, PMCID: PMC4973108.

11) Rolfson DB, Majumdar SR, Tsuyuki RT, Tahir A, Rockwood K. Validity and reliability of the Edmonton Frail Scale. Age Ageing. 2006; 35(5): 526-9. PMID: 16757522.

12) Pialoux T, Goyard J, Lesourd B. Screening tools for frailty in primary health care: a systematic review. Geriatr Gerontol Int. 2012; 12(2): 189-97. doi: 10.1111/j.1447-0594.2011.00797.x. PMID: 22233158.

13) Fit for Frailty. Part 1: Recognition and management of frailty in individuals in community and outpatient setting. Availible from: http://www.bgs.org.uk/campaigns/fff/fff_full.pdf .

14) Hebert R, Raiche M, Dubois MF, Gueye NR, Dubuc N, Tousignant M. Impact of PRISMA, a coordinationtype integrated service delivery system for frail older people in Quebec (Canada): a quasi-experimental study. J Gerontol B Psychol Sci Soc Sci. 2010; 65B: 107-18. doi: 10.1093/geronb/gbp027. PMID: 19414866.

15) Simone PM, Haas AL. Frailty, Leisure Activity and Functional Status in Older Adults: Relationship With Subjective Well Being. Clinical Gerontologist. 2013; 36(4): 275-93. doi: 10.1080/07317115.2013.788114

16) Setiati S, Aryana S, Seto E, Sunarti S, Widajanti N, Dwipa L, et al. Frailty status and its associated factors among indonesian elderly people. Innovation in Aging. 2017; 1(suppl 1): 386. doi: 10.1093/geroni/igx004.1400.

17) Woo J, Goggins W, Sham A, Ho SC. Social determinants of frailty. Gerontology. 2005; 51(6): 402-8. PMID: 16299422.

18) Juma S, Taabazuing M-M, Montero-Odasso M. Clinical Frailty Scale in an Acute Medicine Unit: a Simple Tool That Predicts Length of Stay. Canadian Geriatrics Journal. 2016;19(2):34-39. doi:10.5770/cgj.19.196.

19) Raîche M, Hébert R, Dubois MF. PRISMA-7: a case-finding tool to identify older adults with moderate to severe disabilities. Arch Gerontol Geriatr. 2008; 47(1): 9-18. doi: 10.1016/j.archger.2007.06.004. PMID: 17723247.

20) Saenger ALF, Caldas CP, Raîche M, da Motta LB. Identifying the loss of functional independence of older people residing in the community: Validation of the PRISMA-7 instrument in Brazil. Arch Gerontol Geriatr. 2018; 74: 62-7. doi: 10.1016/j.archger.2017.09.008. PMID: 29031102. 